\title{
Curcumin loaded pH-sensitive hybrid lipid/block copolymer nanosized drug delivery systems
}

Ivelina Jelezova, Elena Drakalska, Denitsa Momekovab , Natalia Shalimova, Georgi

Momekov, Spiro Konstantinov, Stanislav Rangelov, Stergios Pispas

\begin{abstract}
Curcumin is a perspective drug candidate with pleiotropic antineoplastic activity, whose exceptionally low aqueous solubility and poor pharmacokinetic properties have hampered its development beyond the preclinical level. A possible approach to overcome these limitations is the encapsulation of curcumin into nano-carriers, incl. liposomes. The present contribution is focused on feasibility of using hybrid $\mathrm{pH}$-sensitive liposomes, whereby curcumin is entrapped as a free drug and as a water soluble inclusion complex with PEGylated tertbutylcalix[4]arene, which allows the drug to occupy both the phospholipid membranes and the aqueous core of liposomes. The inclusion complexes were encapsulated in dipalmithoylphosphathydilcholine:cholesterol liposomes, whose membranes were grafted with a poly(isoprene-b-acrylic acid) diblock copolymer to confer $\mathrm{pH}$-sensitivity. The liposomes were characterized by DLS, $\zeta$-potential measurements, cryo-TEM, curcumin encapsulation efficacy, loading capacity, and in vitro release as a function of $\mathrm{pH}$. Free and formulated curcumin were further investigated for cytotoxicity, apoptosis-induction and caspase-8, and 9 activation in chemosensitive HL-60 and its resistant sublines HL-60/Dox and HL-60/CDDP. Formulated curcumin was superior cytotoxic and apoptogenic agent vs. the free drug. The mechanistic assay demonstrated that the potent proapoptotic effects of $\mathrm{pH}$-sensitive liposomal curcumin presumably mediated via recruitment of both extrinsic and intrinsic apoptotic pathways in both HL-60 and HL-60/CDDP cells.
\end{abstract}

